\title{
A Review of Biomarkers for Neurodegenerative Disease: Will They Swing Us Across the Valley?
}

Thomas G. Beach

Received: February 15, 2017

(C) The Author(s) 2017. This article is an open access publication

\section{ABSTRACT}

Measures of the severity of cognitive impairment or parkinsonism are the usual endpoints in clinical trials for Alzheimer's disease (AD) and Parkinson's disease (PD), but are critically hampered by their lack of disease sensitivity and specificity. Due to the high failure rate of clinical trials, the rate of regulatory approval for efficacious new drugs has stagnated in the past few decades, with the gap between basic science discovery and clinical application metaphorically termed the "Valley of Death". While the causes for this are probably multiple and complex, the usage of biomarkers as surrogate endpoints, particularly when they are molecularly-specific for the disease, has achieved some success in cancer trials, and it is likely that neurodegenerative disease trials would benefit from the same approach. As dementia and parkinsonism are not disease-specific clinical syndromes, both $\mathrm{AD}$ and PD trials have been flawed by reliance on clinical diagnosis and clinical endpoints. Clinical improvement has been a requirement for

Enhanced content To view enhanced content for this article go to http://www.medengine.com/Redeem/ CAD8F06039796B6E.

T. G. Beach ( $\square)$

Banner Sun Health Research Institute, Sun City, AZ, USA

e-mail: Thomas.Beach@bannerhealth.com regulatory approval, but molecularly-specific biomarkers should improve both diagnostic accuracy and tracking of disease progression, allowing quicker screening of drug candidates. However, even when a molecularly-specific biomarker is found, such as amyloid imaging for $\mathrm{AD}$, it may not reflect the entire extant molecular disease repertoire and may not serve equally well in the different roles of preclinical detection, diagnostic confirmation and surrogate endpoint, necessitating the usage of two, three or more biomarkers, deployed in series or in parallel.

Keywords: Blood; Cerebrospinal fluid; Clinical trial; Diagnosis; Etiology; Pathogenesis; Treatment

\section{RECOGNITION \\ OF AN INADEQUATE RATE OF DISCOVERY OF NEW THERAPEUTIC AGENTS}

Across medical disciplines, we have been presented with a challenge that is both disheartening and provocative. Recognition of a lack of tangible, widespread progress has been openly admitted and graphically portrayed as the "Valley of Death" [1]. Despite an avalanche of data emanating from powerful new technology, 
the production rate of new effective medications has been steadily dropping. Although the Valley concept took root from cancer research, neurodegenerative research is no exception. The major Food and Drug Association (FDA)-approved therapeutic agents for Alzheimer's disease and Parkinson's disease are based on work done three to four decades ago. Current FDA-approved therapies for the primary symptoms of $\mathrm{AD}$ are still largely restricted to cholinergic replacement, an approach that was suggested by human brain tissue studies in the 1970s [2-5]. The only other approach, directed at blocking glutamatergic excitotoxicity (memantine/Namenda), was approved by the FDA in 2003, but the first description of excitotoxicity had been in 1957 [6], and its application to AD was first envisioned in the early 1980s. Similarly, for PD, dopaminergic replacement therapy is still the mainstay, more than 50 years after the discovery of the dopaminergic deficit [7]. Immunotherapy and agents targeting $A \beta$ secretases are undergoing intensive clinical testing but have so far failed to produce convincing results.

Now, our most urgent question has become, "Where have we gone wrong?" The Valley of Death has been variously portrayed as a chasm between biomedical researchers and patients, or between basic science researchers in academia and applied science researchers in the pharmaceutical industry, or between basic science ideas and the hurdles they must cross to regulatory approval. What is clear is that the translation of basic science discoveries to drugs with obvious real-world benefits is becoming less, rather than more, frequent than anyone is comfortable with. Multiple reasons for the gap have been advanced, including the increasingly complex route to FDA approval, regulatory changes leading to a dramatic cost escalation of large clinical trials, the increasing dependence on for-profit corporate involvement, and the realization that many diseases are etiologically heterogeneous [8-10].

Another possible cause has received much less attention but may be much more important. In 2002, the National Dialogue on Cancer, convened to understand why the "war on cancer" was falling short of expectations, concluded that, of the ten most important roadblocks to finding cures for cancer, the single most critical one was inadequate availability of "high-quality, highly characterized human tissues for translational research", and this has also been advanced as an obstacle to finding new therapeutic agents for AD and PD [11]. The comparison of appropriate diseased and control tissue has long been the most direct and most productive route to discovery of new, disease-relevant molecular pathways.

This article is based on previously conducted studies and does not involve any new studies of human or animal subjects performed by the author.

\section{ARE WE TREATING TOO LATE?}

It has long been known, based on autopsy findings of "incidental" plaques, tangles and Lewy bodies in clinically-normal older people, that subjects with $\mathrm{AD}$ and PD must spend decades in a preclinical stage. In the past 10 years, this concept has become increasingly accepted, and characterization of the preclinical state has been seen as a crucial goal. Models of the trajectory of $\mathrm{AD}$ through asymptomatic, mild impairment and overt dementia have progressed from initial autopsy-based work [12-14] to those based on biomarkers measured in vivo $[15,16]$. Combined with the repeated failure of clinical trials of subjects with AD dementia, the increased awareness and acceptance of a continuum between preclinical and manifest disease stages prompted the conclusion that therapy for AD might have a much higher chance for success if it was aimed at slowing or halting progression in the preclinical stage.

In 2011, three consensus panels, jointly sponsored by the National Institute on Aging and the Alzheimer's Association, recommended that $\mathrm{AD}$ be defined and studied in terms of three clinically-defined stages, preclinical, mild cognitive impairment and dementia [17-19]. This was a paradigm shift in that previous expert clinician panels had always considered dementia as an essential part of the definition of $\mathrm{AD}$, while leaving the existence of a preclinical stage in limbo. The panel that most directly 
articulated this paradigm shift was that reported on by Sperling et al. [19]. This panel emphasized that $\mathrm{AD}$ is likely best treated when subjects are in a presymptomatic or preclinical phase, before large-scale neurodegeneration has occurred, and that the definition of this needs to be addressed using evidence from biomarker, epidemiological, and neuropsychological studies. To formalize this shift in thinking, the panel suggested that a re-definition of AD is necessary. Whereas formerly the definition of AD included the presence of dementia, it would be more advantageous and more accurate to realize that $\mathrm{AD}$ is a continuous and gradual pathophysiological disease process, with a long asymptomatic period during which biomarkers might track an individual's otherwise unseen disease progression. However, it was acknowledged that at the present time insufficient longitudinal data exist, preventing an accurate prediction of clinical course from biomarker measures. Ultimately, the concerted results from prospective studies, with measures and interpretation done within a standardized framework encompassing definitions and methodology, are what are hoped to identify biomarker profiles of subjects most likely to benefit from disease-modifying therapies.

\section{BIOMARKERS AS ACCELERANTS FOR CLINICAL TRIALS}

The shift towards trying to prevent AD dementia from happening, rather than trying to cure it after it occurs, had its roots decades ago, with many authors suggesting that $\mathrm{AD}$, like myocardial and cerebral infarctions, might be prevented by appropriate therapy. Again, following this model, it has long been apparent that the measurement of appropriate biomarkers, i.e. blood pressure and blood lipid profile, have been essential to the prevention of heart attack and stroke, and that, with similarly-effective biomarkers, those at risk for $\mathrm{AD}$ dementia could be identified and treated prior to any overt signs or symptoms.

At this point, it is useful to briefly review the definition and evolution of biomarkers. A search of the US National Library of Medicine
(PubMed) shows that the earliest appearance of the term in the title of a publication was in 1980, referring to a blood serum biomarker of breast cancer [20]. Shortly afterwards, the advantages of using biomarkers in trials of cancer [21] and heart disease [22] were widely discussed [23]. By 2001, in order to clarify developing ambiguity, the term was given a consensus definition by an NIH working group [24], stated as follows: "Biological marker (biomarker): A characteristic that is objectively measured and evaluated as an indicator of normal biological processes, pathogenic processes, or pharmacological responses to a therapeutic intervention". Additionally, several possible uses of biomarkers were suggested, including (1) in early-phase clinical trials to establish "proof-of-concept", (2) as a diagnostic tool for those subjects with a disease or an abnormal condition, (3) for staging of disease or classification of the extent of disease, (4) as an indicator of disease prognosis, and (5) for the prediction and monitoring of clinical response to an intervention. Perhaps the most important usage of biomarkers, as a "surrogate endpoint" in clinical trials, was extensively discussed. The usual and definitive proof of the efficacy of a medication, in the opinion of regulatory agencies such as the US FDA, has always been, and will continue to be, "clinical endpoints" that are measures of how a patient "feels, functions or survives". Common clinical endpoints include myocardial infarction, stroke, cancer recurrence and death. A biomarker would qualify as a surrogate endpoint if it predicted clinical benefit. A major advantage of surrogate biomarkers as compared to clinical endpoints is that they are often less expensive, easier to measure and more precise [25].

With respect to $\mathrm{AD}, \mathrm{PD}$ and other neurodegenerative diseases, a previously unrecognized issue has been the relatively low diagnostic accuracy for both of these conditions, particularly at early stages, using only standard neurological examination and investigation [26-29]. It is very likely that many clinical trials done over the past few decades have been underpowered because this was not factored into the predicted effect size, and the major reason for Phase 2 clinical trial failure has been 
low effect size [30]. It is expected that biomarkers will improve diagnostic accuracy and that their deployment to assist trial subject selection will greatly improve trial efficiency [31].

The clinical endpoints have been progression of cognitive impairment or motor dysfunction. These have been suboptimal for rapidly assessing new candidate drugs, as they are nonspecific syndromes that are produced by multiple diseases with differing molecular etiologies. Currently, autopsy studies have shown that most elderly subjects have more than one brain pathology present [32], and that most of the variability in cognitive performance is not accounted for, even by the summation of common histological lesions [33]. Additionally, the rates of change in functional measures are slow, necessitating long and costly clinical trials. These shortcomings are particularly critical for prevention trials, where clinical endpoints might take more than a decade to appear. Efforts should be made, using available clinicopathological data, to identify functional measures that correlate better with the responsible pathological lesions, giving greater disease specificity.

\section{NEUROPATHOLOGICAL VALIDATION IS CRITICAL}

A variety of biomarkers are currently being deployed in $\mathrm{AD}$ prevention and/or treatment trials, including cerebrospinal fluid (CSF) $A \beta$, tau, phosphorylated tau and other neuronal proteins, PET tracers for $A \beta$, tau and glucose uptake, and MR measures of brain atrophy [34-37]. A serious shortcoming for many of these, however, is the lack of validation by neuropathology at autopsy. While the FDA has made autopsy validation a requirement for approval of new PET agents, other imaging modalities as well as biofluid markers have not been forced to meet this standard, and as a result their true accuracy is unknown as they have been validated mostly against the relatively inaccurate clinical diagnosis, or against other unvalidated biomarkers. A second-best approach is to validate new biomarkers against an autopsy-validated biomarker, as has been done by comparing CSF AD biomarkers to PET amyloid imaging [38-40]. There are diminishing returns to this strategy, however, as, if we assume an average $85 \%$ sensitivity and specificity of PET amyloid for neuropathologically-confirmed AD [31], then a biomarker that achieves $80 \%$ sensitivity and specificity against PET amyloid might identify only $68 \%$ of $\mathrm{AD}$ subjects correctly $(0.85 \times 0.80)$.

\section{SUCCESS STORY WITH LIMITATIONS: PET AMYLOID AS A BIOMARKER FOR AD}

PET imaging of brain amyloid deposits has gained wide acceptance as the best modality for this role, with the confidence being primarily due to autopsy validation of the three currently-approved agents [41-43]. Although not explicitly approved for diagnosing dementia due to $\mathrm{AD}$, its usage probably increases clinical diagnostic accuracy by at least $10 \%$ points $[31,44]$. However, it has important limitations. None of the approved agents detects neuropathologically "sparse" neuritic plaque densities, and so amyloid PET may not be the optimal method to select subjects for prevention studies based on anti-amyloid therapeutics, as only those with the relatively heavier "moderate" and "frequent' neuritic plaque densities are amyloid PET-positive. On the other hand, subjects with AD dementia have usually reached a plateau in plaque density, which may limit the ability of an anti-amyloid agent to reduce PET-demonstrated plaque loads. Additionally, until now, amyloid PET has provided mostly a "yes-no" answer to plaque presence and is not useful for disease staging, although SUVr quantitation could be employed and striatal PET amyloid deposits may demonstrate two stages of plaque regional spread, mirroring Thal histopathological plaque staging [45-47]. Perhaps the optimal usage of amyloid PET is for selecting amyloid-positive subjects for secondary prevention or mild cognitive impairment trials in order to exclude subjects without amyloid. The experience with the phase III solanezumab and bapineuzumab trials 
indicated that about $25 \%$ of included subjects were amyloid PET-negative, despite being selected with the traditional neurological criteria from the pre-amyloid imaging era [48]. Perhaps most disappointing has been the disconnect between plaque load reduction and cognitive improvement [49], thus far denying amyloid PET true surrogate status, although the Phase $1 \mathrm{~b}$ aducanamab trial of 125 subjects offers hope of achieving this in subsequent trials [50].

\section{THE NEAR AND FAR FUTURE}

PET imaging for the demonstration of tau pathology in $\mathrm{AD}$, although so far not validated by autopsy (an autopsy-validated study is currently being conducted by Avid), has the great advantage, over any other AD biomarkers, of potentially being able to provide, as would striatal PET amyloid, pathologically-based clinical staging of AD. Flortaucipir (formerly AV-1451) has been shown by several groups [51-53] to demonstrate cognitively-correlated cortical patterns similar to those originally described by Braak [54]. As a surrogate biomarker for clinical AD, Braak staging with tau PET would potentially be the best yet, as autopsy studies have shown that regional brain tau pathology is the strongest correlate of cognitive impairment and disease progression $[55,56]$. Yet some limitations may be foreseen, including a relatively low sensitivity to non-AD tauopathy as well as low-abundance tau pathology, pretangles and other "immature" tau pathology [57-59].

Nascent biofluids methods currently impressing include high-sensitivity assays such as MagQu's detection system utilizing magnetic nanoparticles [60, 61] and "seeding" and "quaking" assays that take advantage of the prion-like properties of pathological tau and alpha-synuclein [62-64]. Early reports of extremely high sensitivity and specificity have to be tempered against the reality that clinical diagnosis has so far been the only gold standard, reducing accuracies of $90 \%$ to less-stellar levels.

Biomarkers for the synucleinopathies have been relatively underperforming as there is as yet no PET agent [65], while CSF alpha-synuclein concentrations broadly overlap with normal [66, 67]. A concerted effort has been made by the Michael J. Fox Foundation (MJFF) to spur the development of a PET diagnostic and a peripheral tissue synuclein-based biomarker. A multi-center group sponsored by the MJFF has provided considerable clarification to the latter field, demonstrating with autopsy-validated tissue that discrepancies in earlier work have been due to idiosyncracies of single-center studies, inadequate definition of disease-specific staining patterns and insufficient training of histopathology readers [68-70]. A current Phase IV observational study, "S4", is being sponsored by the MJFF and will determine the sensitivity and specificity of immunohistochemical staining for pathological alpha-synuclein in colonic, submandibular gland and skin biopsies from 60 PD subjects and 20 controls [71].

Genotype is expected by many to be an especially powerful biomarker, not only in autosomal dominantly-inherited forms of disease and individually strong risk factors but also in terms of composite possession of up to dozens of weaker risk and protective alleles [72]. In large genome-wide studies, the inaccuracy of the clinical diagnostic gold standard has been overcome by huge sample size, but it has been recognized that genomic studies utilizing subjects with a neuropathological diagnosis, because of the more precise molecular phenotyping, may be done with much smaller subject numbers and with a much richer payout in terms of clinicopathological correlations $[73,74]$. Genome-wide association studies have been superb at revealing molecular risk factors, but at present it seems that, at least for neurodegenerative disease, genomic analysis may continue to lack the precision required for individualized medicine.

The eventual bogeyman to contend with, however, will be molecular neuropathological complexity, as neurodegenerative diseases most commonly occur in groups rather than as solo performers [32, 75-78], and this heterogeneity of pathology has also been demonstrated in subjects enrolled in diagnostic trials [79, 80]. Such comorbidity increases with extreme old age, but is still common in subjects in their 70s. 
Successfully detecting and treating one disease may not be completely effective when there are two, three or even four present.

In summary, biomarkers for neurodegenerative disease have come a long way in the past 10 years, but validation against autopsy still occurs too infrequently. Useful single-pathology methods are having an impact on clinical trials and in limited clinical settings, but combinations of pathology are likely to forestall them from reaching their full potential in the near future.

\section{ACKNOWLEDGEMENTS}

No funding or sponsorship was received for this study or publication of this article. The named author meets the International Committee of Medical Journal Editors (ICMJE) criteria for authorship for this manuscript, takes responsibility for the integrity of the work as a whole, and has given final approval for the version to be published.

Disclosures. Thomas G. Beach has served as a consultant to GE Healthcare, Bayer Healthcare, Prothena, Avid Radiopharmaceuticals, Hoffman-LaRoche and Genentech, and is currently doing contracted research for Avid Radiopharmaceuticals and Navidea Biopharmaceuticals.

Compliance with Ethics Guidelines. This article is based on previously conducted studies and does not involve any new studies of human or animal subjects performed by the author.

Data Availability. Data sharing is not applicable to this article as no datasets were generated or analyzed during the current study.

Open Access. This article is distributed under the terms of the Creative Commons Attribution-NonCommercial 4.0 International License (http://creativecommons.org/licenses/ by-nc/4.0/), which permits any noncommercial use, distribution, and reproduction in any medium, provided you give appropriate credit to the original author(s) and the source, provide a link to the Creative Commons license, and indicate if changes were made.

\section{REFERENCES}

1. Butler D. Translational research: crossing the Valley of Death. Nature. 2008;453:840-2.

2. McGeer PL, Eccles JC, McGeer EG. Catecholamine neurons. In: Molecular neurobiology of the mammalian brain. 1987 ; p. 265-8.

3. Davies P, Maloney AJ. Selective loss of central cholinergic neurons in Alzheimer's disease. Lancet. 1976;2:1403.

4. White P, Hiley CR, Goodhardt MJ, et al. Neocortical cholinergic neurons in elderly people. Lancet. 1977;1:668-71.

5. Perry EK, Tomlinson BE, Blessed G, et al. Correlation of cholinergic abnormalities with senile plaques and mental test scores in senile dementia. $\mathrm{Br}$ Med J. 1978;2:1457-9.

6. Olney JW. The toxic effects of glutamate and related compounds in the retina and the brain. Retina. 1982;2:341-59.

7. Hornykiewicz O. Dopamine miracle: from brain homogenate to dopamine replacement. Mov Disord. 2002;17:501-8.

8. LoRusso PM, Schnipper LE, Stewart DJ, et al. Translating clinical trials into meaningful outcomes. Clin Cancer Res. 2010;16:5951-5.

9. Booth CM. Evaluating patient-centered outcomes in the randomized controlled trial and beyond: informing the future with lessons from the past. Clin Cancer Res. 2010;16:5963-71.

10. Stewart DJ, Whitney SN, Kurzrock R. Equipoise lost: ethics, costs, and the regulation of cancer clinical research. J Clin Oncol. 2010;28:2925-35.

11. Beach TG. Alzheimer's disease and the "Valley of Death": not enough guidance from human brain tissue? J Alzheimers Dis. 2013;33(Suppl 1):S219-33.

12. Duyckaerts C, Hauw JJ. Prevalence, incidence and duration of Braak's stages in the general population: can we know? Neurobiol Aging. 1997;18:362-9.

13. Braak H, Braak E. Frequency of stages of Alzheimer-related lesions in different age categories. Neurobiol Aging. 1997;18:351-7. 
14. Ingelsson M, Fukumoto $\mathrm{H}$, Newell KL, et al. Early Abeta accumulation and progressive synaptic loss, gliosis, and tangle formation in AD brain. Neurology. 2004;62:925-31.

15. Jack CR Jr, Knopman DS, Jagust WJ, et al. Hypothetical model of dynamic biomarkers of the Alzheimer's pathological cascade. Lancet Neurol. 2010;9:119-28.

16. Jack CR Jr, Knopman DS, Jagust WJ, et al. Tracking pathophysiological processes in Alzheimer's disease: an updated hypothetical model of dynamic biomarkers. Lancet Neurol. 2013;12:207-16.

17. Albert MS, DeKosky ST, Dickson D, et al. The diagnosis of mild cognitive impairment due to Alzheimer's disease: recommendations from the National Institute on Aging-Alzheimer's Association workgroups on diagnostic guidelines for Alzheimer's disease. Alzheimers Dement. 2011;7:270-9.

18. McKhann GM, Knopman DS, Chertkow H, et al. The diagnosis of dementia due to Alzheimer's disease: recommendations from the National Institute on Aging-Alzheimer's Association workgroups on diagnostic guidelines for Alzheimer's disease. Alzheimers Dement. 2011;7:263-9.

19. Sperling RA, Aisen PS, Beckett LA, et al. Toward defining the preclinical stages of Alzheimer's disease: recommendations from the National Institute on Aging-Alzheimer's Association workgroups on diagnostic guidelines for Alzheimer's disease. Alzheimers Dement. 2011;7:280-92.

20. Paone JF, Waalkes TP, Baker RR, et al. Serum UDP-galactosyl transferase as a potential biomarker for breast carcinoma. J Surg Oncol. 1980;15:59-66.

21. Ellenberg S, Hamilton JM. Surrogate endpoints in clinical trials: cancer. Stat Med. 1989;8:405-13.

22. Wittes J, Lakatos E, Probstfield J. Surrogate endpoints in clinical trials: cardiovascular diseases. Stat Med. 1989;8:415-25.

23. Strimbu K, Tavel JA. What are biomarkers? Curr Opin HIV AIDS. 2010;5:463-6.

24. Biomarkers Definitions Working Group. Biomarkers and surrogate endpoints: preferred definitions and conceptual framework. Clin Pharmacol Ther 2001;69:89-95.

25. Aronson JK. Biomarkers and surrogate endpoints. $\mathrm{Br}$ J Clin Pharmacol. 2005;59:491-4.

26. Sabbagh MN, Shah F, Reid RT, et al. Pathologic and nicotinic receptor binding differences between mild cognitive impairment, Alzheimer disease, and normal aging. Arch Neurol. 2006;63:1771-6.
27. Adler $\mathrm{CH}$, Beach TG, Hentz JG, et al. Low clinical diagnostic accuracy of early vs advanced Parkinson disease: clinicopathologic study. Neurology. 2014;83:406-12.

28. Beach TG, Monsell SE, Phillips LE, et al. Accuracy of the clinical diagnosis of Alzheimer disease at National Institute on Aging Alzheimer Disease Centers, 2005-2010. J Neuropathol Exp Neurol. 2012;71:266-73.

29. Dugger BN, Davis K, Malek-Ahmadi M, et al. Neuropathological comparisons of amnestic and nonamnestic mild cognitive impairment. BMC Neurol. 2015;15:146.

30. Arrowsmith J. Trial watch: Phase II failures: 2008-2010. Nat Rev Drug Discov. 2011;10:328-9.

31. Beach TG, Schneider JA, Sue LI, et al. Theoretical impact of Florbetapir (18F) amyloid imaging on diagnosis of alzheimer dementia and detection of preclinical cortical amyloid. J Neuropathol Exp Neurol. 2014;73:948-53.

32. Beach TG, Adler CH, Sue LI, et al. Arizona study of aging and neurodegenerative disorders and brain and body donation program. Neuropathology. 2015;35:354-89.

33. Boyle PA, Wilson RS, Yu L, et al. Much of late life cognitive decline is not due to common neurodegenerative pathologies. Ann Neurol. 2013;74:478-89.

34. Villemagne VL, Dore V, Bourgeat $\mathrm{P}$, et al. Abeta-amyloid and Tau imaging in dementia. Semin Nucl Med. 2017;47:75-88.

35. Langbaum JB, Fleisher AS, Chen K, et al. Ushering in the study and treatment of preclinical Alzheimer disease. Nat Rev Neurol. 2013;9:371-81.

36. Jack CR Jr, Bennett DA, Blennow $\mathrm{K}$, et al. A/T/N: an unbiased descriptive classification scheme for Alzheimer disease biomarkers. Neurology. 2016;87:539-47.

37. Hoglund K, Kern S, Zettergren A, et al. Preclinical amyloid pathology biomarker positivity: effects on tau pathology and neurodegeneration. Transl Psychiatry. 2017;7:e995.

38. Blennow K, Mattsson N, Scholl M, et al. Amyloid biomarkers in Alzheimer's disease. Trends Pharmacol Sci. 2015;36:297-309.

39. Lewczuk P, Matzen A, Blennow $\mathrm{K}$, et al. Cerebrospinal fluid Abeta42/40 corresponds better than Abeta42 to amyloid PET in Alzheimer's disease. J Alzheimers Dis. 2017;55:813-22. 
40. Morris E, Chalkidou A, Hammers A, et al. Diagnostic accuracy of (18)F amyloid PET tracers for the diagnosis of Alzheimer's disease: a systematic review and meta-analysis. Eur J Nucl Med Mol Imaging. 2016;43:374-85.

41. Clark CM, Pontecorvo MJ, Beach TG, et al. Cerebral PET with florbetapir compared with neuropathology at autopsy for detection of neuritic amyloid-beta plaques: a prospective cohort study. Lancet Neurol. 2012;11:669-78.

42. Curtis C, Gamez JE, Singh U, et al. Phase 3 trial of flutemetamol labeled with radioactive fluorine 18 imaging and neuritic plaque density. JAMA Neurol. 2015;72:287-94.

43. Sabri O, Sabbagh MN, Seibyl J, et al. Florbetaben PET imaging to detect amyloid beta plaques in Alzheimer's disease: phase 3 study. Alzheimers Dement. 2015;11:964-74.

44. Boccardi M, Altomare D, Ferrari C, et al. Assessment of the incremental diagnostic value of florbetapir $\mathrm{F}$ 18 imaging in patients with cognitive impairment: the incremental diagnostic value of amyloid PET with [18F]-florbetapir (INDIA-FBP) study. JAMA Neurol. 2016;73:1417-24.

45. Thal DR, Beach TG, Zanette M, et al. [F]flutemetamol amyloid positron emission tomography in preclinical and symptomatic Alzheimer's disease: Specific detection of advanced phases of amyloid-beta pathology. Alzheimers Dement. 2015;11:975-85.

46. Beach TG, Thal DR, Zanette M, et al. Detection of striatal amyloid plaques with [18F]flutemetamol: validation with postmortem histopathology. J Alzheimers Dis. 2016;52:863-73.

47. Thal DR, Rub U, Orantes $M$, et al. Phases of a beta-deposition in the human brain and its relevance for the development of $\mathrm{AD}$. Neurology. 2002;58:1791-800.

48. Delrieu J, Ousset PJ, Voisin T, et al. Amyloid beta peptide immunotherapy in Alzheimer disease. Rev Neurol (Paris). 2014;170:739-48.

49. Rygiel K. Novel strategies for Alzheimer's disease treatment: an overview of anti-amyloid beta monoclonal antibodies. Indian J Pharmacol. 2016;48:629-36.

50. Sevigny J, Chiao P, Bussiere T, et al. The antibody aducanumab reduces Abeta plaques in Alzheimer's disease. Nature. 2016;537:50-6.

51. Schwarz AJ, Yu P, Miller BB, et al. Regional profiles of the candidate tau PET ligand 18F-AV-1451 recapitulate key features of Braak histopathological stages. Brain. 2016;139:1539-50.
52. Scholl M, Lockhart SN, Schonhaut DR, et al. PET imaging of tau deposition in the aging human brain. Neuron. 2016;89:971-82.

53. Wang L, Benzinger TL, Su Y, et al. Evaluation of tau imaging in staging Alzheimer disease and revealing interactions between beta-amyloid and tauopathy. JAMA Neurol. 2016;73:1070-7.

54. Braak H, Braak E. Neuropathological stageing of Alzheimer-related changes. Acta Neuropathol (Berl). 1991;82:239-59.

55. Nelson PT, Abner EL, Schmitt FA, et al. Modeling the association between 43 different clinical and pathological variables and the severity of cognitive impairment in a large autopsy cohort of elderly persons. Brain Pathol. 2010;20:66-79.

56. Nelson PT, Alafuzoff I, Bigio EH, et al. Correlation of Alzheimer disease neuropathologic changes with cognitive status: a review of the literature. J Neuropathol Exp Neurol. 2012;71:362-81.

57. Marquie M, Normandin MD, Vanderburg CR, et al. Validating novel tau positron emission tomography tracer [F-18]-AV-1451 (T807) on postmortem brain tissue. Ann Neurol. 2015;78:787-800.

58. Lowe VJ, Curran G, Fang P, et al. An autoradiographic evaluation of AV-1451 Tau PET in dementia. Acta Neuropathol Commun. 2016;4:58.

59. Marquie M, Normandin MD, Meltzer AC, et al. Pathological correlations of [F-18]-AV-1451 imaging in non-alzheimer tauopathies. Ann Neurol. 2017;81:117-28.

60. Chiu MJ, Yang SY, Chen TF, et al. New assay for old markers-plasma beta amyloid of mild cognitive impairment and Alzheimer's disease. Curr Alzheimer Res. 2012;9:1142-8.

61. Yang CC, Yang SY, Chieh JJ, et al. Biofunctionalized magnetic nanoparticles for specifically detecting biomarkers of Alzheimer's disease in vitro. ACS Chem Neurosci. 2011;2:500-5.

62. Fairfoul G, McGuire LI, Pal S, et al. Alpha-synuclein RT-QuIC in the CSF of patients with alpha-synucleinopathies. Ann Clin Transl Neurol. 2016;3:812-8.

63. Furman JL, Diamond MI. FRET and flow cytometry assays to measure proteopathic seeding activity in biological samples. Methods Mol Biol. 2017;1523:349-59.

64. Saijo E, Ghetti B, Zanusso G, et al. Ultrasensitive and selective detection of 3-repeat tau seeding activity in Pick disease brain and cerebrospinal fluid. Acta Neuropathol. 2017;133:751-65. 
65. Catafau AM, Bullich S. Non-amyloid PET imaging biomarkers for neurodegeneration: focus on tau, alpha-synuclein and neuroinflammation. Curr Alzheimer Res. 2017;14:169-77.

66. Mollenhauer B, Parnetti L, Rektorova I, et al. Biological confounders for the values of cerebrospinal fluid proteins in Parkinson's disease and related disorders. J Neurochem. 2016;139(Suppl 1):290-317.

67. Parnetti L, Cicognola C, Eusebi P, et al. Value of cerebrospinal fluid alpha-synuclein species as biomarker in Parkinson's diagnosis and prognosis. Biomark Med. 2016;10:35-49.

68. Beach TG, Corbille AG, Letournel F, et al. Multicenter assessment of immunohistochemical methods for pathological alpha-synuclein in autopsied sigmoid colon of Parkinson's disease and control subjects. J Parkinson's Dis. 2016;6:761-70.

69. Corbille AG, Letournel F, Kordower JH, et al. Evaluation of alpha-synuclein immunohistochemical methods for the detection of Lewy-type synucleinopathy in gastrointestinal biopsies. Acta Neuropathol Commun. 2016;4:35.

70. Lee JM, Derkinderen P, Kordower JH, et al. The search for a peripheral biopsy indicator of alpha-synuclein pathology for Parkinson disease. J Neuropathol Exp Neurol. 2017;76:2-15.

71. Visanji NP, Mollenhauer B, Beach TG, et al. The systemic synuclein sampling study: toward a biomarker for Parkinson's disease. Biomark Med. 2017;11:359-68.

72. Reiman EM, Langbaum JB, Fleisher AS, et al. Alzheimer's prevention initiative: a plan to accelerate the evaluation of presymptomatic treatments. J Alzheimers Dis. 2011;26(Suppl 3):321-9.
73. Kouri N, Ross OA, Dombroski B, et al. Genome-wide association study of corticobasal degeneration identifies risk variants shared with progressive supranuclear palsy. Nat Commun. 2015;6:7247.

74. Beecham GW, Dickson DW, Scott WK, et al. PARK10 is a major locus for sporadic neuropathologically confirmed Parkinson disease. Neurology. 2015;84:972-80.

75. Jellinger KA, Attems J. Challenges of multimorbidity of the aging brain: a critical update. J Neural Transm. 2015;122:505-21.

76. Bennett DA, Schneider JA, Arvanitakis Z, et al. Neuropathology of older persons without cognitive impairment from two community-based studies. Neurology. 2006;66:1837-44.

77. Negash S, Bennett DA, Wilson RS, et al. Cognition and neuropathology in aging: multidimensional perspectives from the rush religious orders study and rush memory and aging project. Curr Alzheimer Res. 2011;8:336-40.

78. Schneider JA, Arvanitakis Z, Bang W, et al. Mixed brain pathologies account for most dementia cases in community-dwelling older persons. Neurology. 2007;69:2197-204.

79. Dugger BN, Clark CM, Serrano G, et al. Neuropathologic heterogeneity does not impair florbetapir-positron emission tomography postmortem correlates. J Neuropathol Exp Neurol. 2014;73:72-80.

80. Serrano GE, Sabbagh MN, Sue LI, et al. Positive florbetapir PET amyloid imaging in a subject with frequent cortical neuritic plaques and frontotemporal lobar degeneration with TDP43-positive inclusions. J Alzheimers Dis. 2014;42:813-21. 